\title{
Cytomegalovirus Reactivation in Hematopoietic Stem cell Transplantation managed by Preemptive Treatment with lower dose Valganciclovir
}

\author{
Elif Melis Baloglu Akyol ${ }^{1}$ and Handan Haydaroglu Sahin ${ }^{2 *}$ \\ ${ }^{1}$ MD. Gaziantep University, Faculty of Medicine, Department of Internal Medicine, Gaziantep, Turkey \\ ${ }^{2}$ MD. Assistant Professor, Gaziantep University, Faculty of Medicine, Department of Hematology, Gaziantep, Turkey
}

Received: January 24, 2019; Accepted: February 4, 2019; Published: February 7, 2019

*Corresponding author: Handan Haydaroglu Sahin, Assistant Professor, Gaziantep University, Faculty of Medicine Department of Hematology, 27310, Sahinbey / Gaziantep, TURKEY; Tel : +90 342 3606060/76151; +90 507 4867664; Fax: +90 342 4720718; E-mail: tezhandan@hotmail.com; handanh@ gantep.edu.tr

\begin{abstract}
Objective: The aim of this study was to investigate risk factors for cytomegalovirus (CMV) infection, effect of viral reactivation on hematopoietic stem cell transplantation (HSCT) and efficacy of preemptive treatment using a lower dose valganciclovir for the first time.

Material and Method: The data of 447 patients who underwent HSCT for malignant and non-malignant hematological disorders at a single center from September 2009 to December 2016 were retrospectively evaluated in this study. DNA levels of CMV were routinely tested two days per week for the first 24 months following HSCT, and in case of clinical suspicion after 24 months using the Quantitative Real-Time quantitative polymerase chain reaction (RT-PCR).

Results: Ninety (54.2\%) allogeneic transplant patients, and forty one (14.6\%) autologous transplant patients had CMV reactivation. There was a statistically significant increase in CMV reactivation in the non-myeloablative (NMA) allogeneic transplant group compared to the myeloablative (MA) group at a value of 150-1000 copies/mL CMV-PCR ( $\mathrm{p}=0.002)$. Acute/chronic Graft Versus Host Disease (GVHD) were observed in 30 of allogeneic HSCT patients. 29 of these patients (96.6\%) had CMV antigenemia. There was a significant association between the development of acute/ chronic GVHD, and CMV viremia $(\mathrm{p}=0.001)$. The patients without CMV antigenemia had a higher incidence of mortality at first 100-day, and 365-day in allogeneic transplant group ( $\mathrm{p}=0.022, \mathrm{p}=0.024$, respectively). The 5 -year overall survival (OS) rate was $61 \%$ in the viremia group, and $62 \%$ in the non-viremia group. There was no statistically significant difference between the two groups in terms of 5 -year $0 S$ ( $p=0.551$ ). In patients receiving a lower dose of $900 \mathrm{mg} /$ day valganciclovir treatment due to viremia, CMV disease did not develop and no adverse effect that required the drug to be discontinued was observed.
\end{abstract}

Conclusion: The results conducted that development of GVHD was associated with CMV viremia. Early and late mortality rate were higher in the patients without CMV antigenemia. However; CMV reactivation had no impact on patients' survival post allogeneic and autologous HSCT. Also, this was the first study that conducted preemptive treatment approach in the CMV viremia using a lower dose valganciclovir is safe and effective in HSCT.

Key words: Hematopoietic Stem Cell Transplantation; Cytomegalovirus; Greft versus Host Disease; Valganciclovir

\section{Introduction}

Cytomegalovirus (CMV) reactivation and infection are major complications and associated with increased overall mortality after hematopoietic stem cell transplantation (HSCT) [1]. The incidence of CMV infection increases with the intensity and duration of immunosuppression, and approaches $70 \%$ in allogeneic transplant recipients. 50\% of allogeneic transplant patients developed culture proven CMV infection [2]. Risk factors for CMV reactivation identified such as CMV seropositive donor, type of coditioning regimen, use of $\mathrm{T}$ cell depletion, and graftversus-host disease (GVHD) [3]. Although this complication still remains an important cause of morbidity and mortality in patients undergoing HSCT, the availability of effective antiviral drugs and the implementation of preemptive threapy have led to dramatic improvements in patient outcomes [4]. For an effective preemptive treatment, quick and sensitive diagnostic methods that will detect CMV infection are needed. Quantitative real time polymerase chain reaction (RT-PCR) has replaced the lowsensitivity CMV pp 65 antigenemia test in the determination of viral load during diagnosis. However, with regard to the preemptive treatment decision, there exists no international standardized viral load threshold value. In the literature, various values ranging between 150-5000 copies/ml have been suggested as the viral load threshold to initiate preemptive treatment [5]. Ganciclovir and its pro-drug valganciclovir are being used as the first line treatment in CMV infection. The fact that valganciclovir can be taken orally and possesses an efficacy comparable to ganciclovir shows that it can constitute a good alternative to ganciclovir. The recommended dose of valganciclovir in preemptive therapy is 
$2 \times 900 \mathrm{mg} /$ day [6, 7]. In this study we used a dose of $900 \mathrm{mg} /$ day as preemptive treatment with valganciclovir.

In this retrospective study, we analyzed the records of 442 patients to determine the incidence of and the risk factors for CMV infection in transplant patients, effects of viral reactivation on HSCT, and the efficacy of preemptive treatment using a lower dose valganciclovir for the first time.

\section{Patients and Methods}

\section{Patient population}

A total of 447 patients, including 166 patients who underwent Myeloablative (MA) and non-Myeloablative (NMA) allogeneic HSCT and 281 patients who underwent autologous HSCT due to malignant and non-malignant hematological disorders at Gaziantep University Bone Marrow Transplantation Centre between September 2009 and December 2016 were included in the study. Of patients who underwent allogeneic HSCT, 86 received transplants due to a diagnosis of acute myeloid leukemia (AML), 44 due to Acute Lymphoblastic Leukemia (ALL), 9 due to Non-Hodgkin Lymphoma (NHL), and 27 due to Aplastic Anemia (AA); whereas 91 patients underwent autologous HSCT due to a diagnosis of NHL and 179 due to Multiple Myeloma. Demographic characteristics, underlying diseases, CMV states, conditioning regimens, CMV reactivation and outcomes, symptoms associated with CMV infection, treatment modalities, and survival states were obtained from patient files and carefully recorded. Patient data were presented retrospectively.

\section{CMV antigenemia assay}

DNA levels of CMV were routinely tested two days per week for the first 24 months following HSCT, and in case of clinical suspicion after 24 months using the Real-Time quantitative polymerase chain reaction (RT-PCR) (COBAS AmpliPrep/COBAS TaqMan, Roche Molecular Systems, Inc. USA) method. Detection of CMV PCR values higher than 150 copies $/ \mathrm{ml}$ in whole blood at least once was considered CMV viremia. Severity of CMV viremia was evaluated under three groups based on the serum CMV PCR values: Mild viremia was accepted as 150-1500 copies/ $\mathrm{ml}$, moderate viremia as $1000-10000$ copies $/ \mathrm{ml}$, and severe viremia as more than 10000 copies/ml. CMV monitorization was continued for two months following negative CMV PCR results.

\section{Pre-emptive therapy}

Of the patients detected to have CMV antigenemia, eight were administered $1 \times 5 \mathrm{mg} / \mathrm{kg} /$ day iv Ganciclovir once every 12 hours and 70 were administered $900 \mathrm{mg} /$ day Valganciclovir. For 12 patients with positive CMV DNA levels, the treatment was initiated with Ganciclovir and continued with Valganciclovir. The preemptive treatment was concluded in patients who demonstrated two subsequent negative CMV PCR results.

\section{Statistical analysis}

The chi-square test was used in the comparison of CMV viremia with clinical parameters, and the Log Rank test in the comparison of overall survival and clinical parameters. The
Fischer's exact test was utilized where necessary. The Median test was used in the comparison of quantitative data across groups. Moreover, logistic regression models of Odds ratio (OR) and 95\% confidence intervals (CI) were also calculated. OR (95\% CI) was adjusted for age and gender. A p value below 0.05 was considered statistically significant. Numbers, percentages, medians, means, and standard deviations of the data were computed. SPSS 11.5 (SPSS Inc., Chicago, IL, USA) package program was used in the analysis of data.

\section{Results}

\section{Patients}

Clinical and demographic characteristics of the patients have been presented in Table 1 . A median of $6.5 \times 10^{6}$ (range $4-15 \times 10^{6}$ ) CD34+ stem cells were infused in allogeneic HSCT, whereas a median of $8 \times 10^{6}$ (range 3-24 x106) CD34+ stem cells were infused in autologous HSCT. Of patients who received an allogeneic HSCT from a matched sibling/relative donor, 70 received a conventional MA conditioning regimen and 96 an NMA regimen. Peripheral blood stem cells were used as the graft source in all patients. The median age of the 166 patients who underwent an allogeneic HSCT (101 male/65 female) was 29 (range 15-60 years), and the median age of the 281 patients who underwent an autologous HSCT (160 male/121 female) was 54 (range 17-76 years). Median follow-up time was 23 months (range 3-84 months) for the allogeneic transplant group and 28 months (range 3-88 months) for the autologous transplant group (Table 1).

\section{CMV antigenemia}

The median time of initial detection of serologic CMV PCR positivity in the post transplant period was day 33 for the allogeneic HSCT patient group (range 1-465 days) and day 12 for the autologous transplant group (range 3-55 days). The incidence of CMV viremia was $54.2 \%$ across allogeneic transplant patients and $14.6 \%$ in the autologous HSCT group. Of the patients who demonstrated CMV PCR positivity, 68.7\% (90 patients) were in the allogeneic transplant group (44 MA HSCT and 46 NMA HSCT patients), whereas 41 (31.3\%) were in the autologous transplant group. Of patients who received an allogeneic transplant, $28.3 \%$ manifested mild and $23 \%$ manifested moderate CMV viremia. In the autologous transplant group, $60.9 \%$ patients demonstrated mild viremia (Table 1).

\section{Clinic effects of CMV viremia on Allogeneic HSCT}

At levels of 150-1000 copies/ml, serum CMV PCR levels were determined to be significantly higher for patients who received an NMA conditioning regimen compared to those who received an MA HSCT regimen $(p=0.002)$ (Data not shown). Despite serologic CMV positivity, systemic CMV infections were encountered in only two (1.2\%) allogeneic HSCT patients and in the form of CMV colitis. In allogeneic transplant patients; the median time of thrombocyte engraftment was day 16 for the group detected to have viremia (range 12-63 days) and the median time of neutrophil engraftment was day 15 (range 1121), whereas for the CMV PCR negative group, the median time 
Table 1: Baseline characteristics of patients undergoing hematopoietic stem cell transplantation

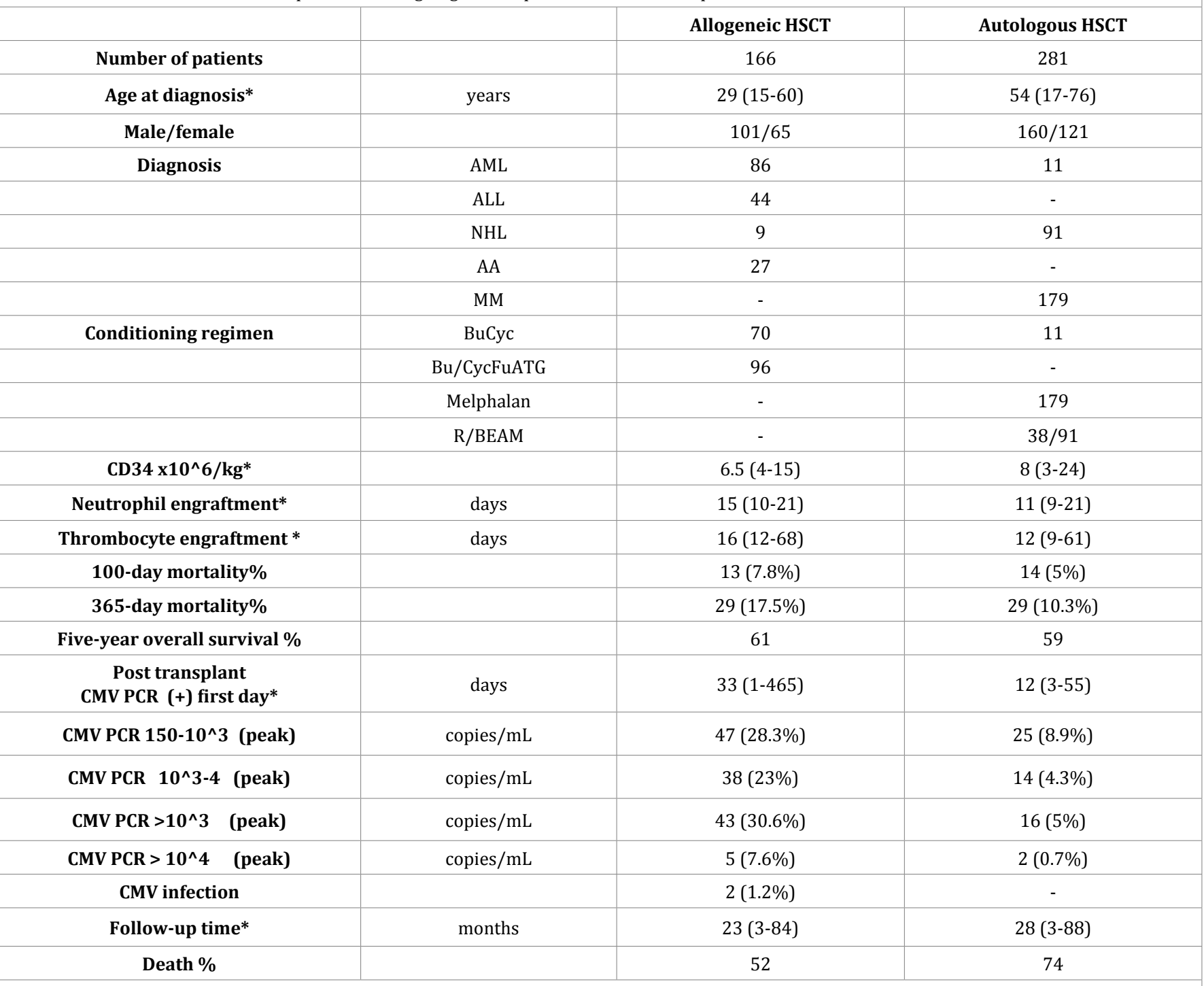

\&: Log rank test, @: Fisher's Exact Test, *: median, \#Median test, CMV: cytomegalovirus, AML: Acute myeloid leukemia, ALL: Acute lymphoblastic leukemia, NHL: Non-Hodgkin lymphoma, AA: Aplastic Anemia, MM: Multiple myeloma, BuCyc: busulfan, cyclophosphamide; Bu/CycFuATG: busulfan, cyclophosphamide, fludarabine, anti-thymocyte globulin; R/BEAM: Rituximab/carmustine, etoposide, cytarabine, melphalan, allogeneic HSCT: Allogeneic hematopoietic stem cell transplantation, Autologous HSCT: Autologous hematopoietic stem cell transplantation

of thrombocyte engraftment was day 15 (range 10-62 days) and the median time of neutrophil engraftment was day 15 (range 10-21). When allogeneic HSCT patients with and without CMV viremia were compared, CMV positivity was found to have no effect on thrombocyte and neutrophil engraftment $(\mathrm{p}=0.905$, $\mathrm{p}=0.479$, respectively). T-cell depletion was done with antithymocyte globulin (ATG) in 50 patients with viremia and 46 patients without viremia. When the two groups were compared; it was found that the use of ATG in the conditioning regimen was not a predisposing factor for developing CMV viremia $(\mathrm{p}=$ 0.533). 30 allogeneic HSCT patients manifested acute/chronic Graft Versus Host Disease (GVHD). 29 of these patients (96.7\%) demonstrated CMV viremia, while one (3.3\%) did not. A strong statistical relationship was determined between CMV viremia and acute/chronic GVHD ( $\mathrm{p}=0.001)$ (Table 2).
In the allogeneic transplant group; mortality was encountered in three patients with CMV viremia (3.3\%) in the early period (first 100 days) and 10 patients (11.1\%) in the late period (365-day-mortality), whereas across CMV PCR negative patients, it was encountered in $10(13.2 \%)$ in the early period and in $19(25 \%)$ in the late period. An evaluation of the relationship between CMV PCR status and mortality revealed that mortality rate in the early and late periods were significantly higher for CMV negative patients $(p=0.022, p=0.024$, respectively). Fiveyear overall survival was similar for the viremia and CMV negative groups with respective rates of $61 \%$ and $62 \%$, and no statistically significant difference was found between the two groups in terms of 5-year OS ( $\mathrm{p}=0.551)$ (Table 2) (Figure 1). 


\begin{tabular}{|c|c|c|c|c|}
\hline & & $\mathrm{CMV}$ viremia $>150$ copies $/ \mathrm{mL}$ & CMV viremia (-) & $P$ value \\
\hline Number of patients & & 90 & 76 & \\
\hline Age at diagnosis* & years & $29(15-60)$ & $32(17-57)$ & \\
\hline Male/female & & $52 / 38$ & $49 / 27$ & \\
\hline Neutrophil engraftment* & days & $15(11-21)$ & $15(10-21)$ & $0.479^{\#}$ \\
\hline Thrombocyte engraftment* & days & $16(12-63)$ & $15(10-62)$ & $0.905^{\#}$ \\
\hline ATG use & & $50(56 \%)$ & $46(60.55)$ & $0.533^{@}$ \\
\hline Acute/Chronic GVHD & & $29(96.7 \%)$ & $1(3.3 \%)$ & $0.001^{@}$ \\
\hline \multirow{4}{*}{ Immunosuppressive use } & CSA or MMF & $54 / 6$ & $70 / 6$ & \\
\hline & $\mathrm{CSA}_{+} \mathrm{PRD}$ & 19 & & \\
\hline & $\mathrm{CSA}_{+} \mathrm{MMF}$ & 8 & & \\
\hline & $\mathrm{CSA}_{+} \mathrm{MMF}_{+} \mathrm{PRD}$ & 3 & & \\
\hline \multirow{3}{*}{ Antiviral treatment } & Ganciclovir & 8 & & \\
\hline & Ganciclovir $\rightarrow$ Valganciclovir & 12 & & \\
\hline & Valganciclovir & 70 & & \\
\hline Antiviral treatment time* & days & $23(10-75)$ & & \\
\hline 100-day mortality $\%$ & & $3(3.3 \%)$ & $10(13.2 \%)$ & $0,022^{@}$ \\
\hline 365-day mortality\% & & $10(11.1 \%)$ & $19(25 \%)$ & $0.024^{@}$ \\
\hline Five-year overall survival \% & & 61 & 62 & $0.551^{\&}$ \\
\hline
\end{tabular}

${ }^{\&}$ Log rank test,*: median, \#: Median test, @Fisher's Exact Test, CMV: cytomegalovirus, ATG: anti-thymocyte globulin, GVHD: graft versus host disease, CSA: Cyclosporine, MMF: mycophenolate mofetil, PRD: prednisolone

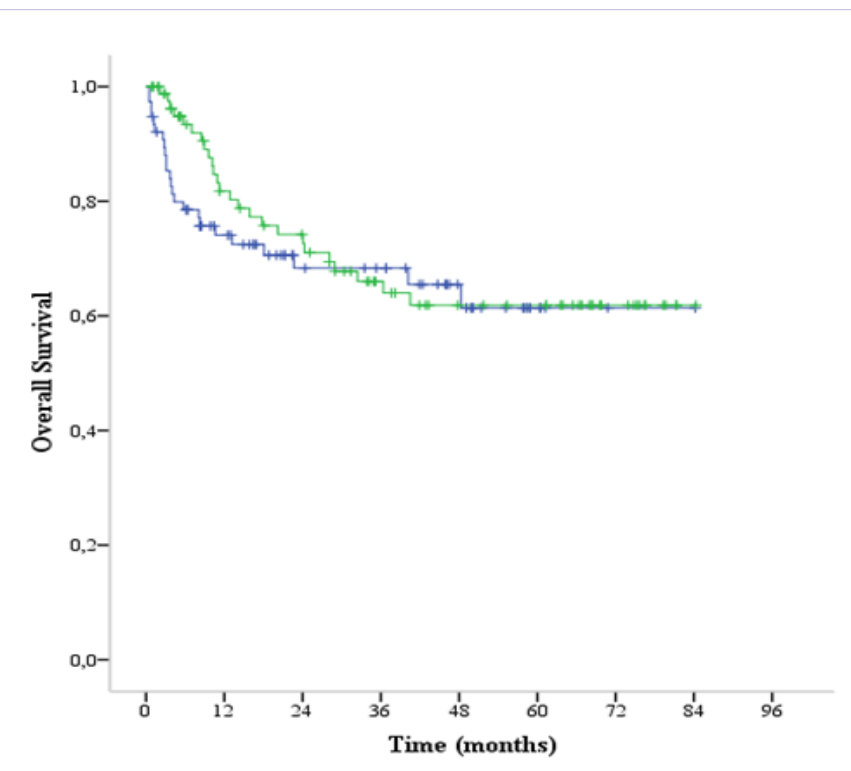

Figure 1: The relationship between CMV values and total overall survival in patients who underwent allogeneic HSCT

Allogeneic HSCT: Allogeneic Hematopoietic Stem Cell Transplantation, CMV: Cytomegalovirus

\section{Clinic effects of CMV viremia on Autologous HSCT}

In the autologous transplant group, 41 patients (14.6\%) were detected to have CMV viremia. The median time of thrombocyte engraftment was day 12 for the group without viremia (range 9-61 days) and the median time of neutrophil engraftment was day 11 (range 10-21), whereas for the CMV-positive group, the median time of thrombocyte engraftment was day 13 (range 9-61 days) and the median time of neutrophil engraftment was day 11 (range 9-21). CMV viremia was found to have no effect on engraftment time ( $\mathrm{p}=0.452, \mathrm{p}=0.866$, respectively).

The conditioning regimens of four autologous HSCT patients with CMV viremia and 37 without viremia involved Rituximab. When the groups with and without CMV viremia were compared with regard to Rituximab use; it was found that Rituximab use was not a risk factor for CMV viremia $(\mathrm{p}=0.474)$. Two patients with CMV viremia received ganciclovir and 34 received valganciclovir therapy. In five cases, the treatment was initiated with ganciclovir and continued with valganciclovir. Patients with CMV viremia received parenteral/oral antiviral therapy for a median of 14 days (range 10-55 days). No symptoms of active infection were encountered in patients detected to have viremia. Mortality was encountered in two patients with CMV viremia (4.9\%) and 12 patients without viremia (5\%) in the early period. Late-term mortality was encountered in four patients in the CMV positive group $(9.6 \%)$ and 25 patients in the negative group (10.4\%). CMV viremia did not have a significant relationship with early or late mortality (both, $p>0.05$ ). 5-year OS was $66 \%$ for the viremia group and $56 \%$ for the group without viremia. CMV reactivation following autologous transplant was found to have no effect on patient survival $(\mathrm{p}=0.660)$ (Table 3$)$. 


\begin{tabular}{|c|c|c|c|c|}
\hline & & CMV viremia $>150$ copies $/ \mathrm{mL}$ & CMV viremia (-) & $P$ value \\
\hline Number of patients & & 41 & 240 & \\
\hline Age at diagnosis* & Years & $55(20-66)$ & $53(17-76)$ & \\
\hline Male/female & & $27 / 14$ & $133 / 107$ & \\
\hline Neutrophil engraftment* & days & $11(9-21)$ & $11(10-21)$ & $0.866^{\#}$ \\
\hline Thrombocyte engraftment* & days & $13(9-61)$ & $12(9-61)$ & $0.452^{\#}$ \\
\hline Rituximab use & & $4(9.8 \%)$ & $37(15.4 \%)$ & $0.474^{@}$ \\
\hline \multirow[t]{3}{*}{ Antiviral treatment } & Ganciclovir & 2 & & \\
\hline & Ganciclovir $\rightarrow$ Valganciclovir & 5 & & \\
\hline & Valganciclovir & 34 & & \\
\hline Antiviral treatment time* & days & $14(10-55)$ & & \\
\hline 100-day mortality\% & & $2(4.9 \%)$ & $12(5 \%)$ & $1.000^{@}$ \\
\hline 365-day mortality\% & & $4(9.6 \%)$ & $25(10.4 \%)$ & $1.000^{\circledR}$ \\
\hline Five-year overall survival \% & & 66 & 56 & $0.660^{\&}$ \\
\hline
\end{tabular}

\section{Discussion}

CMV reactivation and infection constitute one of the significant causes of morbidity and mortality after HSCT. Studies report variable results with regard to the incidence of $\mathrm{CMV}$ viremia in transplant patients. George et al. determined the incidence of viremia as $39.1 \%$ and the incidence of disease as $13 \%$ in allogeneic transplant patients who were treated with a 60\% MA and $40 \%$ Reduced-Intensity Conditioning (RIC) conditioning regimen [8]. Boeckh et al. reported the incidence of CMV disease in allogeneic transplant patients as $17.8 \%$ [9]. The prevalence of CMV viremia in allogeneic HSCT patients is higher compared to the autologous transplant group (1). In the literature, CMV viremia incidence rates following autologous transplant were usually reported as $30-40 \%$. For their 136 autologous transplant patients, Rossini et al. reported the incidence of viremia as $29.4 \%$ and the prevalence of CMV infection as $4.4 \%$ [10]. Another study reported a CMV viremia incidence of $39.6 \%$ [11]. On the other hand, Al-Rawi et al. reported a lower viremia incidence of $17.8 \%$ with the use of prophylactic anti-viral medication [12]. Studies have reported a significant decrease in CMV-related morbidity and mortality in the recent years due to use of prophylactic and preemptive therapies (4). While some studies have reported a decrease in the incidence of viremia and the frequency of infections with the use of prophylactic anti-virals [13], some have reported no effect on incidence, though with lower levels of CMV PCR positivity (12). In this study; the incidence of CMV viremia in allogeneic transplant patients was determined to be higher than that reported in many studies in the literature $(54.2 \%)$ while the incidence of CMV disease was lower (1.2\%), whereas, it was determined to be lower in autologous transplant patients $(14.6 \%)$. The high incidence of CMV viremia may be connected to the low socio-cultural levels of the local people and lack of hygiene; however, the low incidence of CMV disease despite seropositivity suggests that valganciclovir is effective as a preemptive treatment.

The role of immunosuppressive, which is used for $\mathrm{T}$ cell depletion in allogeneic HSCT, as risk factors for CMV viremia, is disputed. Certain studies showed increased CMV reactivation due to the use of ATG for T cell depletion [14, 15]. Using RIC HSCT, Alemtuzumab, and Acute GVHD have been identified as risk factors for CMV viremia (13). While some studies reported lower CMV incidences in patients treated with an NMA conditioning regimen [16-18], others have mentioned increased incidence rates [19]. On the other hand, $\mathrm{Oh}$ and colleagues did not determine a relationship between CMA viremia and the use of NMA or MA conditioning regimens [20]. In our study, 76 patients received an MA and 90 an NMA conditioning regimen. 31 patients (34.4\%) who received an NMA conditioning regimen demonstrated significantly higher rates of weak CMV PCR positivity compared to MA transplant patients and this situation may be linked to the remaining infected lymphocytes in the NMA conditioning regimen. T cell depletion was done using ATG in six MA patients and in all NMA patients. Of the 96 total patients where ATG was used, 50 demonstrated CMV viremia, while 46 did not. ATG use was not determined to be a risk factor for CMV viremia ( $p=0.533)$.

GVHD is considered the most prominent cause of morbidity and mortality after HSCT. Even in fully HLA matched transplants, its incidence is approximately $30 \%$ despite immunosuppressive treatment [21]. Valadkhani et al. determined an acute GVHD incidence of $71 \%$ and a grade II-IV acute GVHD incidence of 57\% in allogeneic transplant patients. $14 \%$ of patients with GVHD of Grade 2 or higher demonstrated high levels of CMV positivity; having GVHD of Grade 2 or higher was identified as a strong risk factor for CMV infections ( $p=0.035$ ) [22]. Similarly, Lin et al. reported that the presence of acute GVHD of Grade 3 or higher 
resulted in a 2.98-fold increase in CMV reactivation (19). Broers et al. identified symptomatic CMV disease as an independent risk factor for acute GVHD, but did not determine a significant relationship between chronic GVHD and CMV reactivation [23]. In light of current data, the relationship between CMV and GVHD can be best described as bidirectional. While GVHD and its treatment increase the risk of a CMV infection, based on current data, the presence of a CMV infection may in turn be connected to developing GVHD [24]. In our study, 30 allogeneic transplant patients manifested GVHD. Of these, 29 (96.7\%) had viremia. In accordance with the literature, our results confirm that GVHD is a strong risk factor for CMV viremia $(p=0.001)$.

When the effects of CMV viremia during the transplantation process are considered, the analyses reveal that there is no consensus in the literature on its relationship with mortality and total overall survival (OS). George et al. defined CMV viremia as a risk factor that negatively affects five-year-OS (13). Another study reported viral load, CMV-specific T cell immune failure, and lymphopenia as a cause of mortality in the late period (9). Lin et al. reported that CMV viremia was not a factor that affected mortality and survival (19). In our study, mortality was encountered in three (3.3\%) allogeneic transplant patients with CMV viremia in the early period and in $10(11.1 \%)$ in the late period, whereas it was encountered in $10(13.2 \%)$ without viremia in the early period and in $19(25 \%)$ in the late period. In allogeneic HSCT patients, early and late term mortality was determined to be significantly lower in the group with CMV viremia $(p=0.022, p=0.024$, respectively). Results of the study suggest that this is connected to the activation of the immune system by CMV-specific T cells.

In the recentyears, developing treatment strategies to prevent complications related to CMV viremia and infection have become a popular research topic across Bone Marrow Transplantation centers. In a study that compared low-dose valganciclovir (900 $\mathrm{mg}$ /day) prophylaxis to $1800 \mathrm{mg} /$ day valganciclovir preemptive treatment, valganciclovir prophylaxis was not determined to be superior to the preemptive treatment strategy. The same study determined an increase in the use of hematopoietic growth factor in the patient group that received valganciclovir prophylaxis (12). Another study found that long-term use of antiviral medications increased mortality caused by reasons other than relapse due to drug-induced neutropenia, and recommended the use of preemptive treatment instead of prophylaxis as antiviral therapy (23). Preemptive treatment with ganciclovir is associated with an increase in the risk of neutropenia, thrombocytopenia, and invasive aspergillus, and this restricts its use [25]. Compared to ganciclovir, valganciclovir appears to be a more favorable option in preemptive treatment with its oral use and lower side effect profile. The recommended dose for preemptive treatment is $2 \times 900$ mg/day (10). In our study, patients with CMV PCR levels higher than 150 copies/ml either received $900 \mathrm{mg} /$ day valganciclovir or $5 \mathrm{mg} / \mathrm{kg}$ ganciclovir twice per day as preemptive treatment. 70 of the 90 allogeneic HSCT patients with CMV viremia and 34 of the 40 autologous transplant patients only received valganciclovir therapy at low doses. In our study, the durations of preemptive treatment that the patients received were median 23 days (range 10-75 days) in the allogeneic HSCT group and median 14 days (range 10-55 days) in the autologous HSCT group, with required treatment times comparable to those in the literature. In our study, a lower dose of $900 \mathrm{mg} /$ day was preferred in preemptive treatment with valganciclovir compared to other studies in the literature and this provided an effective treatment. Patients who received valganciclovir did not manifest cytopenia and renal toxicity that would require the medication to be stopped. This situation may be explained by the lower Valganciclovir dosage preferred in preemtpive treatment. It was found that the two patients who manifested CMV disease had received ganciclovir, whereas the patients who had received valganciclovir did not develop CMV disease. Valganciclovir appears to be more advantageous than other anti-CMV agents due to its oral use, not requiring hospitalization, and consequently, leading to decreased risk of nosocomial infection. This is the first study in the literature that has administered preemptive treatment with a low $900 \mathrm{mg} /$ day dose of valganciclovir.

In summary, CMV reactivation still appears to be a significant problem, especially after allogeneic HSCT. This study determined the use of an NMA conditioning regimen prior to the transplantation and the presence of acute/chronic GVHD as strong risk factors for CMV reactivation. CMV viremia decreases early and late mortality, however, has no effect on patient survival after transplant. In preemptive treatment, administering a lower dose Valganciclovir to the transplant patients appears to be an effective and safe treatment approach.

\section{Acknowledgement}

This study had no specific funding

\section{Conflict of interest}

There is not any conflict of interest

\section{Ethical Approval}

Written voluntary consent forms were obtained from the enrolled patients. This study was conducted after obtaining an approval from Gaziantep University Medical Faculty Medical Ethics Committee, which was dated 25.01.2017 and numbered 2017/10.

\section{References}

1. Wingard JR, Chen DY, Burns WH, Fuller DJ, Braine HG, Yeager AM, Kaiser $\mathrm{H}$, et al. Cytomegalovirus infection after autologous bone marrow transplantation with comparison to infection after allogeneic bone marrow transplantation. Blood. 1988;71(5): 1432-1437.

2. Hebart H, Jahn G, Sinzger C, Kanz L, Einsele H. CMV Infection in Bone Marrow and Solid Organ Transplant Patients in the Era of Antiviral Prophylaxis. Herpes. 2000;7(1):13-17.

3. Rubie H, Attal M, Campardou AM, Gayet-Mengelle C, Payen C, Sanguignol F, Calot JP, et al. Risk factors for cytomegalovirus infection in BMT recipients transfused exclusively with seronegative blood products. Bone Marrow Transplant. 1993;11(3):209-214. 
4. Huang YT, Su Y, Kim SJ, Nichols P, Burack D, Maloy M, Giralt S, et al. Cytomegalovirus Infection in Allogeneic Hematopoietic Cell Transplantation Managed by the Preemptive Approach: Estimating the Impact on Healthcare Resource Utilization and Outcomes. Biol Blood Marrow Transplant. 2018; pii: S1083-8791(18)30745-6. Doi: 0.1016/j.bbmt.2018.11.012

5. Solano C, de la Cámara R, Vázquez L, López J, Giménez E, Navarro D. Cytomegalovirus Infection Management in Allogeneic Stem Cell Transplant Recipients: a National Survey in Spain. J Clin Microbiol. 2015;53(8):2741-2744. Doi: 10.1128/JCM.01057-15

6. McIntosh M, Hauschild B, Miller V. Human cytomegalovirus and transplantation: drug development and regulatory issues. J Virus Erad. 2016;2(3):143-148.

7. Razonable RR. Antiviral drugs for viruses other than human immunodeficiency virus. Mayo Clin Proc. 2011;86(10): 1009-1026. Doi: $10.4065 / \mathrm{mcp} .2011 .0309$

8. George B, Kerridge IH, Gilroy N, Huang G, Hertzberg MS, Bradstock KF, Gottlieb DJ, et al. A risk score for early cytomegalovirus reactivation after allogeneic stem cell transplantation identifies low-, intermediate-, and high-risk groups: reactivation risk is increased by graft-versushost disease only in the intermediate-risk group. Transpl Infect Dis 2012;14(2):141-148. Doi: 10.1111/j.1399-3062.2011.00706.x

9. Boeckh M, Leisenring W, Riddell SR, Bowden RA, Huang ML, Myerson D, Stevens-Ayers T, et al. Late cytomegalovirus disease and mortality in recipients of allogeneic hematopoietic stem cell transplants: importance of viral load and T-cell immunity. Blood. 2003;101(2):407414.

10. Rossini F, Terruzzi E, Cammarota S, Morini F, Fumagalli M, Verga L, Elli E, et al. Cytomegalovirus infection after autologous stem cell transplantation: incidence and outcome in a group of patients undergoing a surveillance program. Transpl Infect Dis. 2005;7(34):122-125.

11. Hebart H, Schröder A, Löffler J, Klingebiel T, Martin H, Wassmann B, Gerneth F, et al. Cytomegalovirus monitoring by polymerase chain reaction of whole blood samples from patients undergoing autologous bone marrow or peripheral blood progenitor cell transplantation. J Infect Dis. 1997;175(6): 1490-1493.

12. Boeckh M, Nichols WG, Chemaly RF, Papanicolaou GA, Wingard JR, Xie $\mathrm{H}$, Syrjala KL, et al. Valganciclovir for the prevention of complications of late cytomegalovirus infection after allogeneic hematopoietic cell transplantation: a randomized trial. Ann Intern Med. 2015;162(1):110. Doi: $10.7326 / \mathrm{M} 13-2729$

13. George B, Pati N, Gilroy N, Ratnamohan M, Huang G, Kerridge I, Hertzberg M, et al. Pre-transplant cytomegalovirus (CMV) serostatus remains the most important determinant of CMV reactivation after allogeneic hematopoietic stem cell transplantation in the era of surveillance and preemptive therapy. Transpl Infect Dis. 2010;12(4): 322-329. Doi: 10.1111/j.1399-3062.2010.00504.x

14. Schmidt-Hieber M, Schwarck S, Stroux A, Ganepola S, Reinke P, Thiel E, Uharek L, et al. Immune reconstitution and cytomegalovirus infection after allogeneic stem cell transplantation: the important impact of in vivo T cell depletion. Int J Hematol. 2010;91(5):877-885. Doi: 10.1007/s12185-010-0597-6

15. Busca A, Passera R, Pini M, Zallio F, Dellacasa C, Audisio E, Giaccone L, et al. The use of ATG abrogates the antileukemic effect of cytomegalovirus reactivation in patients with acute myeloid leukemia receiving grafts from unrelated donors. Am J Hematol. 2015;90(6):E117-21. Doi: 10.1002/ajh.23998

16. Ringdén O, Erkers T, Aschan J, Garming-Legert K, Le Blanc K, Hägglund $\mathrm{H}, \mathrm{Omazic} \mathrm{B}$, et al. A prospective randomized toxicity study to compare reduced-intensity and myeloablative conditioning in patients with myeloid leukaemia undergoing allogeneic haematopoietic stem cell transplantation. J Intern Med. 2013;274(2):153-162. Doi: 10.1111/ joim.12056

17. Junghanss C, Boeckh M, Carter RA, Sandmaier BM, Maris MB, Maloney DG, Chauncey T, et al. Incidence and outcome of cytomegalovirus infections following nonmyeloablative compared with myeloablative allogeneic stem cell transplantation, a matched control study. Blood. 2002;99(6):1978-1985.

18. Nakano N, Kubota A, Tokunaga M, T Itoyama, T Makino, S Takeuchi, Y Takatsuka \& A Utsunomiya, et al. High incidence of CMV infection in adult T-cell leukemia/lymphoma patients after allogeneic hematopoietic stem cell transplantation. Bone Marrow Transplant. 2014;49(12):1548-1549. Doi: 10.1038/bmt.2014.204

19. Lin HC, Han SM, Hwang WL, Cheng-Wei Chou, Kuang-Hsi Chang, ZhiYuan Shi, Chieh-Lin Jerry Teng, et al. Cytomegalovirus Infection and Treatment in Allogeneic Hematopoietic Stem Cell Transplantation: A Retrospective Study from a Single Institution in an Endemic Area. Turk J Haematol. 2017;34(2):159-166. Doi: 10.4274/tjh.2016.0225

20. Oh SJ, Lee KH, Lee JH, Choi SJ, Kim WK, Lee JS, Kim MN, et al. The risk of cytomegalovirus infection in non-myeloablative peripheral stem cell transplantation compared with conventional bone marrow transplantation. J Korean Med Sci. 2004;19(2):172-176.

21. Provan D, Singer CRJ, Baglin T, Dokal I. Oxford Handbook of Haematology (3rd Ed). Oxford, Oxford University Press. 2009.

22. Valadkhani B, Kargar M, Ashouri A, Hadjibabaie M, Gholami K, Ghavamzadeh A. The risk factors for cytomegalovirus reactivation following stem cell transplantation. J Res Pharm Pract. 2016;5(1):6369. Doi: 10.4103/2279-042X.176554

23. Broers AE, van Der Holt R, van Esser JW, Gratama JW, Henzen-Logmans $\mathrm{S}$, Kuenen-Boumeester V, Löwenberg B, et al. Increased transplantrelated morbidity and mortality in $\mathrm{CMV}$-seropositive patients despite highly effective prevention of CMV disease after allogeneic T-celldepleted stem cell transplantation. Blood. 2000;95(7): 2240-2245.

24.Chan ST, Logan AC. The clinical impact of cytomegalovirus infection following allogeneic hematopoietic cell transplantation: Why the quest for meaningful prophylaxis still matters. Blood Rev. 2017;31(3):173183. Doi: $10.1016 /$ j.blre.2017.01.002

25. Einsele H, Hebart H, Kauffmann-Schneider C, Sinzger C, Jahn G, Bader $\mathrm{P}$, Klingebiel $\mathrm{T}$, et al. Risk factors for treatment failures in patients receiving PCR-based preemptive therapy for CMV infection. Bone Marrow Transplant. 2000;25(7):757-763. 